\title{
DIFFUSION AND THE TORSION PARAMETER
}

\author{
ALEX MCNABB $^{1}$ and GRANT KEADY ${ }^{2}$
}

(Received 3 June 1991; revised 15 May 1992)

\begin{abstract}
The parameters describing the trapping kinetics of a linear model for diffusion, in solids involving a captured immobile phase of the diffusing entity, can be determined by measuring mean residence times for matter in the systems and evaluating the exponents for the final exponential decay rates of the diffusing entity from various shaped solids. The mean residence time for matter in a given region can be expressed in terms of a "torsion parameter" $S$ which in the case of Dirichlet boundary conditions and cylindrical geometries, coincides with the torsional rigidity of the cylinder. The final decay rate is given by the first eigenvalue $\mu$ of a Helmholtz problem. Expressions and inequalities are derived for these parameters $S$ and $\mu$ for general linear boundary conditions and for geometries relevant to diffusion experiments.
\end{abstract}

\section{Introduction}

The linear partial differential equations

$$
\alpha \frac{\partial C}{\partial t}-D \Delta^{2} C=-a C+b w=-\frac{\partial w}{\partial T} \quad \text { in } \quad \Omega \times(0, T) ;
$$

model diffusion of a substance which interacts linearly with an immobile phase in a homogeneous region. The dependent variable $C$ is a measure of concentration and diffusion potential, $\alpha$ is the mass storage capacity per unit volume and $D$ is the constant of proportionality between mass flux per unit area and the gradient of diffusion potential normal to the area. The second dependent variable $w$ measures the mass per unit volume of substance trapped in the immobile phase

${ }^{1}$ Department of Mathematics, Massey University, Palmerston North, New Zealand.

${ }^{2}$ Mathematics Department, University of Western Australia, Nedlands, 6009 Western Australia.

(C) Australian Mathematical Society, 1994, Serial-fee code 0334-2700/94 
and the constants $a$ and $b$ characterise the linear trapping kinetics.

In many such systems, particularly relating to diffusion in solids, the parameters $\alpha, D, a, b$ must be inferred from measurements made outside of the region $\Omega$, and so must be related to measurements of $q(t)$ the flux of chemical through $\partial \Omega$ the boundary of $\Omega$, and measurements of $C_{a}(t)$ the concentrations of $C$ of diffusing substance controlling the boundary conditions on $\partial \Omega$ at time $t$. The mass flux $q(t)$ and the accumulated output $Q(t)$ are given by

$$
q(t)=-\int_{\partial \Omega} D \frac{\partial C}{\partial n}, \quad Q(t)=\int_{0}^{t} q(s) d s .
$$

We assume a general linear boundary condition of mixed form:

$$
C+\beta \frac{\partial C}{\partial n}=C_{a} \quad \text { on } \quad \partial \Omega .
$$

If $\Omega$ is saturated by a constant boundary concentration $C_{0}$, then at equilibrium, $C=C_{0}$ and $w=a C_{0} / b$ in $\Omega$. Suppose now that $C$ and $w$ are allowed to decay to zero by setting $C_{a}$ to zero, and $Q(t)$ is measured throughout the decay process. The increasing mass $Q(t)$ of substance evolved after time thas a limiting value $Q(\infty)$ equal to the initial mass content of substance in $G$ and given by

$$
Q(\infty)=V\left(\alpha C_{0}+w_{0}\right)=V(\alpha+a / b) C_{0}=V \alpha^{*} C_{0},
$$

where $\alpha^{*}=\alpha+a / b$ and $V=|\Omega|$, the volume of $\Omega$.

If $\Omega$ is a thin slab of thickness $d$ with $x$ measuring distance through it, and if the ambient concentration $C_{a}$ is maintained constant outside $x=O$ and zero outside the other face at $x=d$, then a steady flux $q$ through the slab given by

$$
q=D \frac{\partial C}{\partial n}=\frac{D C_{a}}{d+2 \beta}
$$

will eventually be attained and driven by a concentration distribution,

$$
C=\frac{C_{a}(d+\beta-x)}{d+2 \beta} .
$$

We see that measurements of $q$ as a function of $d$, or $d$ as a function of $q$ can be used to estimate $D$ and $\beta$. There remains the question of how to identify the other parameters $\alpha, a$ and $b$ in (1.11) and any answer to this must involve a study of transient responses of the system. 
If $C=C_{0}$ at $t=0$ in $\Omega$ when the region is transferred to a zero concentration environment, the mean residence time $\tilde{t}$ for the chemical in $\Omega$ is given by

$$
\tilde{t}=\int_{0}^{\infty} \frac{t q(t)}{Q(\infty)} d t=\int_{0}^{\infty}\left[1-\frac{Q(t)}{Q(\infty)}\right] d t=\int_{\Omega} \frac{\alpha \Phi+\Psi}{Q(\infty)},
$$

where

$$
\Phi=\int_{0}^{\infty} C d t \text { and } \Psi=\int_{0}^{\infty} w d t
$$

The functions $\Phi$ and $\Psi$ are solutions of a Poisson problem.

The integration of (1.1) with respect to time give a linear relationship between $\Phi$ and $\Psi$, and a Poisson equation for $\Phi$ :

$$
-a \Phi+b \Psi=w_{0}=\frac{a C_{0}}{b}, \quad \text { and } \quad D \Delta^{2} \Phi \equiv\left(\alpha+\frac{a}{b}\right) C_{0},
$$

and the same treatment of (1.3) gives a boundary condition for $\Phi$ :

$$
\Phi+\beta \frac{\partial \Phi}{\partial n}=0 \text { on } \quad \partial \Omega
$$

If $u_{\beta}$ is the solution of

$$
\nabla^{2} u_{\beta}=-1 \quad \text { in } \quad \Omega, \quad u_{\beta}+\beta \frac{\partial u_{\beta}}{\partial n}=0 \quad \text { on } \quad \partial \Omega,
$$

and

$$
S_{\beta}=\int_{\Omega} u_{\beta}
$$

we find that

$$
\tilde{t}=\frac{\alpha^{*} S_{\beta}}{D V}+\frac{a}{b^{2} \alpha^{*}} \quad \text { where } \quad \alpha^{*}=\alpha+\frac{a}{b} .
$$

It may be assumed that $D$ and $\beta$ are known from steady flux experiments mentioned earlier and so (1.4), (1.12) determine $\alpha^{*}$ and $a / b^{2}$. A third equation needed to separately identify $\alpha, a$ and $b$, is provided by the exponent $\lambda$ of the final exponential decay made of $q(t)$. If $\mu$ is the first eigenalue of the Helmholtz problem,

$$
\nabla^{2} v+\mu v=0 \quad \text { in } \quad \Omega, \quad v+\beta \frac{\partial v}{\partial n}=0 \quad \text { on } \quad \partial \Omega,
$$

then

$$
\lambda(\lambda-b) \alpha-\lambda a=D \mu(\lambda-b)
$$


In cases of supposed simple diffusion and $a$ and $b$ not too large, we may regard (1.12) as a test for hidden trapped phases. The limiting case for $a$ and $b$ very large is degenerate. In this case the diffusing and trapped populations, $C$ and $w$, are in equilibium and $w=a C / b$. Since measurements of $w$ cannot be made from outside $\Omega$, the system behaves like one of simple diffusion and is indistinguishable from the case where $\alpha$ is replaced by $\alpha^{*}$ in (1.1) and $b w-a C$ is set to zero. In the case $\beta$ zero and $\Omega$ an infinitely long cylinder, $S_{0}$ given by $(\mathrm{P}(0), 1.11)$ is the torsional rigidity of the cylinder and expressions for it may be found in engineering literature for many cross-sectional shapes. (See Higgins [1] for a historical survey of the problem.)

The solution $u_{\beta}$ of the Poisson problem $(\mathrm{P}(\beta))$ also has relevance to freezing problems (McNabb and Wake [7]). When the energy change in a freezing problem is predominantly latent heat of a phase change, $u_{m}$, the greatest value of $u$ in $\Omega$ gives the relative time for freezing of variously shaped regions $\Omega$ in the sense that the ratio $u_{m} / u_{m}^{*}$ of the greatest values of $u$ and $u^{*}$ the solutions of $(\mathrm{P}(\beta))$ in regions $\Omega$ and $\Omega^{*}$ respectively, is equal to the ratio $t_{f} / t_{f}^{*}$ of the times $t_{f}$ and $t_{f}^{*}$ for freezing of the regions $\Omega$ and $\Omega^{*}$.

Exact solutions for problems $(\mathrm{P}(\beta), \mathrm{H}(\beta))$ when $\beta$ is not zero or when $\Omega$ is three dimensional, are hard to find in the literature. $S$ and $\mu$ are easily calculated numerically for two dimensional problems, particularly when $\beta$, the boundary parameter, is zero. For non-zero $\beta$ and three dimensional problems the calculations, while tractable, are more difficult. There are important simple formulae for the solution $u_{\beta}$ and the first eigenvalue $\mu$ of $\mathrm{H}(\beta)$ when $\Omega$ is an infinite slab or circular cylinder and we give new ones for infinite cylinders of rectangular and equilateral triangular cross-section. But these need to be supplemented by correction factors for the finite aspects of experimental geometries and by asymptotic approximations for large $\beta$ values.

In Section 2 we present formulae for the separable variable cases when $\Omega$ is a finite cylinder of rectangular and circular cross-section, which express $u_{\beta}$ as deviations from the simple slab and infinite cylinder cases. These cases have practical value in diffusion studies.

In Section 3, boundary value problems are given for generating the terms of simple asymptotic power series in $(1 / \beta)$ for $u_{\beta}$ and $S_{\beta}$. The first three terms of the asymptotic series for $u_{\beta}$ are:

$$
u_{\beta}=\beta \frac{|\Omega|}{|\partial \Omega|}+u_{\infty}-\frac{1}{\beta} \int_{\partial \Omega} u_{\infty}^{2} d s+\mathrm{O}\left(\frac{1}{\beta^{2}}\right)
$$


where $u_{\infty}$ is the solution of

$$
\nabla^{2} u_{\infty}+1=0 \quad \text { in } \quad \Omega, \quad \frac{\partial u_{\infty}}{\partial n}=-\frac{|\Omega|}{|\partial \Omega|}, \quad \int_{\partial \Omega} u_{\infty} d s=0 \quad \text { on } \quad \partial \Omega .
$$

The boundary value problem $\mathrm{P}(\infty)$ has a simple quadratic polynomial solution when $\Omega$ is a cylinder or box, and even when it is an equilateral triangle. This leads to a simple polynomial solution of $\mathrm{P}(\beta)$ for this region which extends the known solution of $\mathrm{P}(0)$ to general $\beta$. There is even a simple exact solution for the problem $\mathrm{H}(\beta)$ when $\Omega$ is an equilateral triangle.

\section{Separable variable solutions}

The Poisson problem $\mathrm{P}(\beta)$ and the Helmholtz problem $\mathrm{H}(\beta)$ have simple solutions of one independent variable $r$ of the form

$$
u=\frac{a^{2}-r^{2}+2 \beta a}{2 N}, \quad v=\frac{J_{p}(r \sqrt{\mu})}{r^{p}},
$$

where $2 p=N-2$, when $\Omega$ is a sphere in $N$ dimensions of radius $a$. For these cases

$$
\begin{aligned}
& u_{m}=\frac{a^{2}+2 \beta a}{2 N}, \quad \frac{S}{V}=\frac{a^{2}}{N(N+2)}+\frac{\beta a}{N} \\
& \text { and } \quad(p \beta-a) J_{p}(a \sqrt{\mu})=\beta a \sqrt{\mu} J_{p}^{\prime}(a \sqrt{\mu})
\end{aligned}
$$

These are the primary formulae for parameter identification, but need to be supplemented by correction terms for finite geometries. In the cases of rectangular and cylindrical polar coordinates, separable variable techniques yield series solutions of the Poisson problem which give the truncation corrections to the basic formulae (2.2). The derivations are standard (for details see McNabb and Wake [6]) except perhaps for the final formulation as a series of dimensional corrections.

If $\Omega$ is brick shaped and bounded by planes $x= \pm a, y= \pm b, z= \pm c$ the 'torsion function' $u$ for general $\beta$ is given by the expression:

$$
\begin{aligned}
u= & \frac{a^{2}-x^{2}}{2}+\beta a-\sum_{p=1}^{\infty} \frac{a^{2} A_{p}^{1}}{X_{p}^{2}} \frac{\cosh \left(X_{p} y / a\right) \cos \left(X_{p} x / a\right)}{\left[\cosh \left(X_{p} b / a\right)+(\beta / a) X_{p} \sinh \left(X_{p} b / a\right)\right]} \\
& -\sum_{p=1}^{\infty} \sum_{q=1}^{\infty} \frac{c^{2} A_{p}^{1} A_{q}^{2} \cosh \left(Z_{p q} z / c\right) \cos \left(X_{p} x / a\right) \cos \left(Y_{q} y / b\right)}{Z_{p q}^{2}\left[\cosh Z_{p q}+(\beta / c) Z_{p q} \sinh Z_{p q}\right]}
\end{aligned}
$$


where $X_{p}, Y_{q}$ are the roots of the equations,

$$
\cos X_{p}=(\beta / a) X_{p} \sin X_{p}, \quad \cos Y_{q}=(\beta / b) Y_{q} \sin Y_{q},
$$

and $A_{p}^{1}, A_{q}^{2}$ and $Z_{p q}$ are defined as:

$$
\begin{gathered}
A_{p}^{1}=\frac{2 \sin X_{p}}{X_{p}\left[1+(\beta / a) \sin ^{2} X_{p}\right]}, \quad A_{q}^{2}=\frac{2 \sin Y_{q}}{Y_{q}\left[1+(\beta / b) \sin ^{2} Y_{q}\right]}, \\
\frac{Z_{p q}^{2}}{c^{2}}=\frac{X_{p}^{2}}{a^{2}}+\frac{Y_{q}^{2}}{b^{2}} .
\end{gathered}
$$

The greatest value $u_{m}$ of $u$ is at $x=y=z=0$ and is given by

$$
\begin{aligned}
u_{m}= & \frac{a^{2}}{2}+\beta a-\sum_{p=1}^{\infty} \frac{a^{2} A_{p}^{1}}{\left[\cosh \left(X_{p} b / a\right)+(\beta / a) X_{p} \sinh \left(X_{p} b / a\right)\right] X_{p}^{2}} \\
& -\sum_{p=1}^{\infty} \sum_{q=1}^{\infty} \frac{c^{2} A_{p}^{1} A_{q}^{2}}{Z_{p q}^{2}\left[\cosh Z_{p q}+(\beta / c) Z_{p q} \sinh Z_{p q}\right]},
\end{aligned}
$$

and the generalised torsional rigidity $S$ is given by

$$
\begin{aligned}
& \frac{S}{V}=a^{2}\left[\frac{1}{3}+\frac{\beta}{a}\right]-\frac{a^{3}}{b} \sum_{p=1}^{\infty} \frac{2 \sin ^{2} X_{p}}{X_{p}^{5}\left[1+(\beta / a) \sin ^{2} X_{p}\right]\left[\operatorname{coth}\left(X_{p} b / a\right)+(\beta / a) X_{p}\right]} \\
& -c^{2} \sum_{p=1}^{\infty} \sum_{q=1}^{\infty} \frac{\sin ^{2} X_{p} \sin ^{2} Y_{q}}{X_{p}^{2} Y_{q}^{2} Z_{p q}^{3}\left[1+(\beta / a) \sin ^{2} X_{p}\right]\left[1+(\beta / a) \sin ^{2} Y_{q}\right]\left[\operatorname{coth} Z_{p q}+(\beta / c) Z_{p q}\right]}
\end{aligned}
$$

The Helmholtz problem $\mathrm{H}(\beta)$ in the same brick shaped region has the solution:

$$
v=\cos \left(X_{1} \frac{x}{a}\right) \cos \left(Y_{1} \frac{y}{b}\right) \cos \left(Z_{1} \frac{z}{c}\right), \quad \mu=\left(\frac{X_{1}}{a}\right)^{2}+\left(\frac{Y_{1}}{b}\right)^{2}+\left(\frac{Z_{1}}{c}\right)^{2},
$$

where $X_{1}, Y_{1}, Z_{1}$ satisfy equations of the form (2.4).

There are two useful formulae for cylinders, one for coin shaped, and a second for pencil-like regions.

If $\Omega$ is a coin shaped region of radius $a$ and thickness $2 b$, the Poisson problem has a solution.

$$
u(r, z)=\sum_{n=1}^{\infty} R_{n}(r) \cos \left(Z_{n} z / b\right), \quad 0 \leq r \leq a, \quad|z| \leq b,
$$


where $Z_{n}$ is the $n$-th root of

$$
\cos Z_{n}=(\beta / b) Z_{n} \sin Z_{n}
$$

$R_{n}(r)$ is given by,

$$
R_{n}(r)=\frac{A_{n} b^{2}}{Z_{n}^{2}}\left\{1-\frac{I_{0}\left(Z_{n} r / b\right)}{\left[I_{0}\left(Z_{n} \mu\right)+(\beta / b) I_{1}\left(Z_{n} \mu\right)\right]}\right\}
$$

and $A_{n}$ and $\mu$ are defined by

$$
A_{n}=\frac{2 \sin Z_{n}}{Z_{n}\left(1+(\beta / b) \sin ^{2} Z_{n}\right)}, \quad \mu=\frac{a}{b} \geq 1 .
$$

In the limit as the coin radius $a$ goes to infinity the series (2.9) tends to the simple slab formulas (2.1) with $N=1$. Formula (2.11) may be rewritten as

$$
u(r, z)=\frac{b^{2}-z^{2}}{2}+\beta b-\sum_{n=1}^{\infty} \frac{b^{2} A_{n}}{Z_{n}^{2}} \frac{I_{0}\left(Z_{n} r / b\right) \cos \left(Z_{n} z / b\right)}{\left[I_{0}\left(Z_{n} \mu\right)+(\beta / b) Z_{n} I_{1}\left(Z_{n} \mu\right)\right]},
$$

so that $u_{m}$, the greatest value of $u$, is given by

$$
u_{m}=\frac{b^{2}}{2}+\beta b-b^{2} \sum_{n=1}^{\infty} \frac{2 \sin Z_{n}}{Z_{n}^{3}\left[1+(\beta / b) \sin Z_{n}\right]\left[I_{0}\left(Z_{n} \mu\right)+(\beta / b) Z_{n} I_{1}\left(Z_{n} \mu\right)\right]},
$$

and the $S$ value satisfies

$$
\frac{S}{V}=\frac{b^{2}+3 \beta b}{3}-4 b^{2} \sum_{n=1}^{\infty} \frac{\sin ^{2} Z_{n} I_{1}\left(Z_{n} \mu\right)}{\mu^{2} Z_{n}^{6}\left[1+(\beta / b) \sin ^{2} Z_{n}\right]\left[I_{0}\left(Z_{n} \mu\right)+(\beta / b) Z_{n} I_{1}\left(Z_{n} \mu\right)\right]}
$$

The corresponding 'pencil' formulae for a cylinder of radius $a$ and height $2 b(\mu=a / b \leq 1)$ are

$$
u(r, z)=\sum_{n=1}^{\infty} f_{n}(z) J_{0}\left(q_{n} \frac{r}{a}\right), \quad 0 \leq r \leq a, \quad|z| \leq b,
$$

where $q_{n}$ is the $n$-th root of

$$
J_{0}\left(q_{n}\right)=(\beta / a) q_{n} J_{1}\left(q_{n}\right)
$$

the functions $f_{n}(z)$ are given by

$$
f_{n}(z)=a^{2} \frac{B_{n}}{q_{n}^{2}}\left\{1-\frac{\cosh q_{n} z}{\left[\cosh \left(q_{n} / \mu\right)+(\beta / a) q_{n} \sinh \left(q_{n} / \mu\right)\right]}\right\},
$$


and $B_{n}$ and $\mu$ are defined by

$$
B_{n}=\frac{2}{q_{n} J_{1}\left(q_{n}\right)\left(1+\left(\beta^{2} / a^{2}\right) q_{n}^{2}\right)}, \quad \mu=\frac{a}{b} .
$$

The torsion function is now given by

$$
\begin{aligned}
u(r, z)= & \left(a^{2}-r^{2}+2 \beta a\right) / 4 \\
& -\sum_{n=1}^{\infty} \frac{2 a^{2} \cosh \left(q_{n} z / a\right) J_{0}\left(q_{n} r / a\right)}{q_{n}^{3} J_{1}\left(q_{n}\right)\left(1+\left(\beta^{2} / a^{2}\right) q_{n}^{2}\right)\left[\cosh \left(q_{n} / \mu\right)+(\beta / a) q_{n} \sinh \left(q_{n} / \mu\right)\right]}
\end{aligned}
$$

and the alternative expressions for $u_{m}$ and $S / V$ are

$$
\begin{aligned}
u_{m}= & \left(a^{2}+2 \beta a\right) / 4 \\
& -2 a^{2} \sum_{n=1}^{\infty} \frac{1}{q_{n}^{3} J_{1}\left(q_{n}\right)\left(1+\left(\beta^{2} / a^{2}\right) q_{n}^{2}\right)\left[\cosh \left(q_{n} / \mu\right)+(\beta / a) q_{n} \sinh \left(q_{n} / \mu\right)\right]}, \\
\frac{S}{V}= & \left(a^{2}+4 \beta a\right) / 8 \\
& -8 a^{2} \sum_{n=1}^{\infty} \frac{\mu \sinh \left(q_{n} / \mu\right)}{q_{n}^{6}\left(1+\left(\beta^{2} / a^{2}\right) q_{n}^{2}\right)\left[\cosh \left(q_{n} / \mu\right)+(\beta / a) q_{n} \sinh \left(q_{n} / \mu\right)\right]} .
\end{aligned}
$$

The corresponding formulae for $V$ and $\mu$ associated with the problem $\mathrm{H}(\beta)$ are

$$
V=J_{0}\left(q_{1} \frac{r}{a}\right) \cos \left(Z_{1} \frac{z}{b}\right), \quad \mu=\frac{Z_{1}^{2}}{b^{2}}+\frac{q_{1}^{2}}{a^{2}},
$$

where $Z_{1}$ and $q_{1}$ are given by (2.10) and (2.17) respectively.

\section{Asymptotic approximations and the equilateral triangle solution}

When the boundary $\partial \Omega$ of $\Omega$ is sufficiently smooth, the solution $u$ of the problem $\mathrm{P}(\beta)$ can be expressed in the asymptotic series form:

$$
u \sim \frac{\beta|\Omega|}{\partial \Omega}+u_{\infty}+\frac{1}{\beta} u_{1}+\frac{1}{\beta^{2}} u_{2}+\ldots
$$


The functions $u_{\infty}, u_{i}$ are solutions of the differential equations

$$
\nabla^{2} u_{\infty}+1=0, \quad \nabla^{2} u_{i}=0 \quad \text { for } \quad i \geq 1, \quad \text { in } \Omega,
$$

and on the boundary $\partial \Omega$, they satisfy the following conditions:

$$
\begin{gathered}
\frac{\partial u_{\infty}}{\partial n}=-\frac{|\Omega|}{|\partial \Omega|}, \quad \frac{\partial u_{1}}{\partial n}=-u_{\infty}, \quad \frac{\partial u_{i}}{\partial n}=-u_{i-1} \quad \text { for } i>1 ; \\
\int_{\partial \Omega} u_{\infty}=\int_{\partial \Omega} u_{i}=0 .
\end{gathered}
$$

(See Keady and McNabb (1992)). We see that

$$
u=\frac{\beta|\Omega|}{|\partial \Omega|}+u_{\infty}+\mathrm{O}\left(\frac{1}{\beta}\right) \text { for large } \beta,
$$

where $u_{\infty}$ is defined by

$$
\nabla^{2} u_{\infty}+1=0 \quad \text { in } \quad \Omega, \quad \frac{\partial u_{\infty}}{\partial n}=-\frac{|\Omega|}{|\partial \Omega|}, \quad \int_{\partial \Omega} u_{\infty}=0 \quad \text { on } \quad \partial \Omega .
$$

Integration of (3.1) over the domain $\Omega$ gives an asymptotic expansion for $S_{\beta}$, and a variational argument given in Keady and McNabb (1992) gives the useful inequality,

$$
\frac{\beta|\Omega|^{2}}{|\partial \Omega|}+\Sigma_{\infty}+\frac{1}{\beta} \Sigma_{1} \leq S_{\beta} \leq \frac{\beta|\Omega|^{2}}{|\partial \Omega|}+\Sigma_{\infty}
$$

where

$$
\Sigma_{\infty}=\int_{\Omega} u_{\infty}, \quad \Sigma_{1}=\int_{\Omega} u_{1}=-\int_{\partial \Omega} u_{\infty}^{2}
$$

The quadratic polynomial

$$
u_{\infty}=c_{0}-\sum_{j=1}^{n} c_{j} x_{j}^{2}, \quad 2 \sum_{j=1}^{n} c_{j}=1,
$$

solves problem $(\mathrm{P}(\infty))$ for many simple domains.

For example, if $\Omega$ is a brick in $n$ dimensions with sides $\left|x_{i}\right|=a_{i}$, we find

$$
c_{i}=\frac{K}{2 a_{i}}, \quad c_{0}=\frac{K}{6}\left(\sum_{i=1}^{n} a_{i}\right)+\frac{K^{2}}{3} \quad \text { where } \quad \frac{1}{K}=\sum_{i=1}^{n} \frac{1}{a_{i}},
$$


and the function

$$
S_{\infty}=\int_{\Omega} u_{\infty}=\frac{K^{2} V}{3} .
$$

For a circular cylinder of radius $a$ and height $h$,

$$
\begin{aligned}
& u_{\infty}=\frac{a h}{24(a+h)^{2}}\left(3 a^{2}+9 a h+h^{2}\right)-\frac{h r^{2}}{4(a+h)}-\frac{a z^{2}}{2(a+h)}, \\
& S_{\infty}=\frac{5}{24} \pi \frac{a^{4} h^{3}}{(a+h)^{2}} .
\end{aligned}
$$

When $\beta=0$, it is well known (Milne-Thompson [8, page 166]) that the torsion function for the equilateral triangle $\Omega$, with sides of length $2 a$, is

$$
u=U_{0}=\frac{1}{4 \sqrt{3 a}} x(x-\sqrt{3}(y+a))(x+\sqrt{3}(y-a)) .
$$

Thus $u_{m}$, the maximum value, is assumed at the centroid $\left(x_{c}, 0\right)$ where

$$
u_{m}=a^{2} / 9, \quad x_{c}=a / \sqrt{3},
$$

and the torsional rigidity $S_{0}$ is given by

$$
S_{0}=\int_{\Omega} u d v=\frac{\sqrt{3} a^{4}}{20} .
$$

Problem $P(\infty)$ has a solution of the form (3.7) in this triangle given by

$$
u_{\infty}=\frac{x_{c}^{2}}{2}-\frac{1}{4}\left(\left(x-x_{c}\right)^{2}+y^{2}\right) \quad \text { for which } \quad S_{\infty}=\int_{\Omega} u_{\infty}=\frac{a^{4}}{4 \sqrt{3}} .
$$

Surprisingly, this expression leads to a simple exact solution for the general problem $\mathrm{P}(\beta)$. The function

$$
u_{\beta}=\frac{c_{0} u_{0}+\beta u_{\infty}+\beta^{2}(|\Omega| /|\partial \Omega|)+\beta c_{1}}{c_{0}+\beta}
$$

is a solution of the Poisson equation for any constants $c_{0}, c_{1}$ also satisfies

$$
u_{\beta}+\beta \frac{\partial u_{\beta}}{\partial n}=u_{\beta}-\beta \frac{\partial u_{\beta}}{\partial x}=0 \quad \text { on } \quad x=0 \quad \text { when } \quad c_{0}=\frac{a}{\sqrt{3}}, \quad c_{1}=\frac{a^{2}}{6}
$$

and so by symmetry $u_{\beta}$ given by (3.16) is the solution of $\mathrm{P}(\beta)$. 
The value of $u_{m}(\beta)$ found by evaluating $u_{\beta}$ at the centroid is

$$
u_{m}(\beta)=\frac{a\left(2 a^{2}+6 \sqrt{3} a \beta+9 \beta^{2}\right)}{18(a+\sqrt{3} \beta)},
$$

and the function $S_{\beta}=\int_{\Omega} u_{\beta}$ is given by

$$
\begin{aligned}
S_{\beta} & =\frac{c_{0} S_{0}+\beta S_{\infty}+\beta^{2}\left(|\Omega|^{2} /|\partial \Omega|\right)+\beta c_{1}|\Omega|}{c_{0}+\beta} \\
& =\frac{\beta|\Omega|^{2}}{|\partial \Omega|}+S_{0}+\frac{\beta}{c_{0}+\beta}\left(S_{\infty}-S_{0}\right) .
\end{aligned}
$$

The Helmholtz problem $\mathrm{H}(\beta)$ also has a simple exact solution for the equilateral triangle. The function

$$
\begin{aligned}
& v=4 \sin (a+b y) \sin \left[a+\frac{b}{2}(\sqrt{3}-\sqrt{3} x-y)\right] \\
& \times \sin \left[a+\frac{b}{2}(\sqrt{3}+\sqrt{3} x-y)\right]+A \\
&=\sin (a+b \sqrt{3} x+b y)+\sin (a-b \sqrt{3} x+b y)-\sin (a+\sqrt{3} b-2 b y) \\
&+A-\sin (3 a+\sqrt{3} b)
\end{aligned}
$$

satisfies the Helmholtz equation when

$$
A=\sin (3 a+\sqrt{3} b) \text { and } \mu=4 b^{2} .
$$

The requirement that on $y=0$,

$$
\begin{aligned}
v-\beta \frac{\partial v}{\partial y} & =[2 \sin a-2 \beta b \cos a] \cos (b \sqrt{3} x) \\
& \quad+\sin (a+\sqrt{3} b)+2 \beta b \cos (a+\sqrt{3} b) \\
& =0,
\end{aligned}
$$

is satisfied when

$$
t=\tan a=\beta b \quad \text { and } \quad\left(2 t^{2}-1\right) \tan (\sqrt{3} t / \beta)=3 t .
$$

Symmetry considerations imply $v+\beta(\partial v / \partial n)=0$ on the other two sides, and so if $t_{1}$ is the smallest positive solution of (3.24),

$$
\mu=4 t_{1}^{2} / \beta^{2} \text {. }
$$




\section{Conclusion}

The parameters of our simple model for diffusion with linear trapping kinetics can be evaluated by measurements of steady fluxes through slabs and transient experiments measuring flux decay rates and mean residence times for simple geometries. The geometric dependence of the mean residence time is given by a parameter $S$ which, in the case of infinite cylinders and Dirichlet boundary conditions, is the torsional rigidity parameter of elasticity theory. $S$ is easily calculated numerically for two dimensional problems, particularly when $\beta$, the boundary parameter, is zero. For this latter case, the library routine Dø3EAF is used in Keady and $\mathrm{McNabb}$ [3]. For non-zero $\beta$ and three dimensional problems the calculations, while tractable, are more difficult. Analytical series solutions are available for finite cylinders of circular and rectangular cross-section to give truncation corrections for the infinite cylinder formulae. The simple exact solutions for non-zero $\beta$ for the equilateral triangle are useful for program testing and studying solution properties of the problems $\mathrm{P}(\beta)$ and $\mathrm{H}(\beta)$. When $\beta$ is large, the asymptotic inequalities and estimates are needed.

\section{Acknowledgement}

This paper was completed while Grant Keady held a research fellowship at the University of Waikato while on leave without-pay from the University of Western Australia. The support of the New Zealand U.G.C. is gratefully acknowledged.

\section{References}

[1] H. T. J. Higgins, “A comprehensive review of Saint Venant's torsion problem”, Am.J. Phys. 10 (1942) 248-259.

[2] G. Keady and A. McNabb, "Some explicit solutions of $-\Delta w=1$ with zero boundary data", Department of Mathematics Research Report No. 42, University of Western Australia, Dec 1988.

[3] G. Keady and A. McNabb, "The torsion problem in a lens: a case-study in symbolicnumeric computation using SENAC", Department of Mathematics Research Report No. 191, University of Waikato, Hamilton, New Zealand, 1989.

[4] G. Keady and A. McNabb, "The elastic torsion problem: solutions in some convex domains", N.Z.J. Maths (1993) in press. 
[5] G. Keady and A. McNabb, "Functions with constant Laplacian satisfying homogeneous Robin boundary conditions", I.M. A.J. Appl. Math. (1993) in press.

[6] A. McNabb and G. C. Wake, "Some explicit solutions of $-\Delta w=1$ with Robin boundary data", Occasional publication series No. 17, Massey University, Department of Mathematics and Statistics, Palmerston North, New Zealand, 1989.

[7] A. McNabb and G. C. Wake, "Heat conduction and finite transition times between steadystates", I. M. A. J. Appl. Math. 47 (1991) 193-206.

[8] L. M. Milne-Thompson, Antiplane elastic systems (Springer-Verlag, Berlin, 1962). 